\title{
SCATTER IN THE METRICS OF SACCADES AND PROPERTIES OF THE COLLICULAR MOTOR MAP
}

\author{
A. J. van Opstal* and J. A. M. van Gisbergen \\ Department of Medical Physics and Biophysics, University of Nijmegen, Geert Grooteplein Noord 21, \\ 6525 EZ Nijmegen, The Netherlands
}

(Received 2 September 1988; in revised form 4 January 1989)

\begin{abstract}
Saccades, elicited by an identical visual stimulus in repeated trials, exhibit a certain amount of amplitude and direction scatter. The present paper illustrates how this scatter may be used to discern various properties of the subsystem that determines the metrics of a saccade. It is found in humans that scatter along the eccentricity axis is consistently more pronounced than along the direction axis. The ratio of amplitude scatter and direction scatter is approximately constant for all target positions tested. In addition, the absolute amount of scatter increases roughly linearly with target eccentricity but docs not depend on target direction. We have explored whether these findings may reflect noisy variations in the neural representation of the saccade vector at the level of the collicular motor map. There are good reasons to assume that the motor map, at least in the monkey, (1) is organized in polar coordinates, (2) has a nonhomogeneous (roughly logarithmic) representation of saccade amplitude and (3) is anisotropic in nature (Robinson, 1972; Ottes, Van Gisbergen \& Eggermont, 1986; Van Gisbergen, Van Opstal \& Tax, 1987). To account for the intertrial variability in saccades, we have slightly extended an existing model for the collicular role in the coding of saccade metrics (Van Gisbergen et al., 1987) by allowing small variations in both the total amount and the location of the collicular population activity. We discuss how such noisy variations at the level of the motor map would be expressed in the metrics of saccadic responses and consider alternative models which could explain our data.
\end{abstract}

Saccade metrics Saccade variability Ensemble coding model Coding in polar coordinates Human

\section{INTRODUCTION}

\section{Control of saccade amplitude and direction}

How the saccadic control system specifies the metrics of an impending saccade is still a matter of debate. A variety of behavioural experiments, carried out in the recent past, have led to quite different ideas about the subsystem specifying the metrical properties of saccades (Becker \& Jürgens, 1979; Van Gisbergen, Robinson \& Gielen, 1981; Ottes et al., 1984; Deubel, 1987).

Electrophysiological evidence shows that the Superior Colliculus $(S C)$ is heavily involved in the generation of saccade amplitude $(R)$ and direction $(\Phi)$. Recordings from single cells in the deeper layers of the $S C$ demonstrate that these neurons are active just prior to saccades occurring within a limited range of amplitudes and directions specific for each cell: the so-called movement field (Sparks, Holland \& Guthrie, 1976; Sparks \& Mays, 1980). In addition, it has

*To whom correspondence should be addressed. been shown that electrical stimulation yields saccades at short latencies (Robinson, 1972) the metrical properties of which correspond with the movement field of cells nearby the stimulating electrode (Schiller \& Stryker, 1972). Recently, we have proposed a quantitative model for the role of the $S C$ in the generation of saccadic eye movements which incorporates the idea, first expressed by Sparks et al. (1976) and McIlwain $(1976,1982)$, that the execution of the saccadic eye movement depends upon the summed contributions of all recruited cells in the population (Van Gisbergen et al., 1987; Van Opstal \& Van Gisbergen, 1989; see below).

So far, the ensemble coding model is fully deterministic in the sense that it does not have any noise in its subsystems. The work of Deubel (1987) suggests, however, that a detailed analysis of the variability in the metrics of saccadic responses, which can be observed in repeated identical trials, may help to develop the model further. Deubel (1987) noticed that saccade scatter along the eccentricity $(R)$ axis is more 
pronounced than along the direction $(\Phi)$ axis. He pointed out that this finding is interesting in itself since it indicates that part of the saccadic system is organized in a polar $(R, \Phi)$ coordinate system. In the case of independent componentrelated $(x, y)$ subsystems one would rather expect a circular symmetrical distribution of saccade vectors. In this paper we have investigated whether a further quantitative analysis of saccade variability can throw more light on the subsystem thought to underly the generation of saccade metrics and furthermore, whether such a study can help to distinguish among various schemes that have proposed distinct mechanisms for amplitude and direction coding (Becker \& Jürgens, 1979; Van Gisbergen, Van Opstal \& Schoenmakers, 1985; Van Gisbergen et al., 1987; Deubel, 1987). We therefore have tested the implications of a slightly extended version of the ensemble coding model against behavioural data in four human subjects.

Variability in saccade endpoints reflects both noisy variations in the precision of presaccadic fixation and inaccuracies in the saccadic response itself. We have applied a procedure which removes the effect of fixation inaccuracy (see Methods) to ensure that our saccade scatter data truly reflect variability in the programming of the saccade vector rather than peculiarities in fixation. The results, obtained from human subjects, appear to confirm and to extend Deubel's basic finding. Part of these results has been presented elsewhere in a preliminary form (Van Opstal \& Van Gisbergen, 1987a; Van Gisbergen \& Van Opstal, 1987).

\section{METHODS}

\section{Experimental procedures}

Subjects, all male with normal vision, were seated in complete darkness at $57 \mathrm{~cm}$ from a tangent screen on which a bright round target spot $\left(5 \mathrm{~cd} / \mathrm{m}^{2}, 0.5 \mathrm{deg}\right)$ was rear-projected. Our two main subjects (JVG and RJB) were required to make up to 290 saccades to each of various target positions in separate sessions. In the $R$-series experiments, target direction was kept constant at $225 \mathrm{deg}$, and amplitude $(R)$ was varied in subsequent sessions as follows: $2,5,10$, $20,30,40$ and $50 \mathrm{deg}$. In $\Phi$-series experiments, amplitude was set at $20 \mathrm{deg}$ and target directions $(\Phi)$ were: $90,180,200,225,270$ and $315 \mathrm{deg}$. Two other subjects (CLB and WBS) were tested similarly but less extensively (see Tables $2-4$ in Discussion section).
A typical session consisted of 6-8 sequences, each containing 36 test trials to a fixed target position. In order to reduce the occurrence of predictive drift (Kowler \& Steinman, 1979a,b; see also below) 10 control trials, with target directions diametrically opposite to the testtarget direction, were inserted randomly in the sequence. For target eccentricities below $30 \mathrm{deg}$ the stimulus step started at the primary position. In these cases the control target had the same eccentricity as the test target. In the large-amplitude experiments, fixation and target spots were positioned diametrically opposite to the primary position. The eccentricity of the control target in these experiments was at $10 \mathrm{deg}$ with respect to the eccentric fixation position. In all experiments, the target jumped to the peripheral location after a random fixation time (800$1400 \mathrm{msec}$ ). The subject was asked to refixate the peripheral target as fast and as accurately as possible. Subjects were repeatedly urged to abstain from predictive tracking. In all subjects eye movements of the left eye were measured with the search-coil technique, to ensure a high signal-to-noise ratio and linearity over a considerable range (Collewijn, Van der Mark \& Jansen, 1975).

\section{Data-selection criteria}

The following criteria were used in order to select first-saccadic responses from test trials for further analysis: latency had to exceed the value of $100 \mathrm{msec}$, which has been reported to be the lower limit for purely visually-elicited saccades (Kalesnykas \& Hallett, 1987). In addition, response gain had to exceed 0.5 ; saccade direction had to be within $\pm 15 \mathrm{deg}$ from the direction of the stimulus and, finally, the initial fixation position was required to be within 2 standard deviations from the mean. In general, a total of less than $3 \%$ of the data had to be rejected based on these criteria. It should be noted that saccade scatter data always refer to first-saccadic reponses in test trials only.

Despite the fact that the target appeared at the same location on the screen in 36 out of 46 trials (see above), no systematic trend was observed with time in either response gain, latency, normalized amplitude of retinal error after the first saccade or mean saccade velocity (defined as the ratio of saccade amplitude and duration) in the course of the experiment (see Results, Fig. 7), 
Two sources of variability in saccade endpoints

We wish to distinguish between two sources of variability which could contribute to the noisy variations of the saccade endpoints:

(1) Fixation noise. When the subject is asked to foveate the target he will generally do this only with finite accuracy (about $0.5 \mathrm{deg}$ ). Involuntary drift towards the expected stimulus location may aggravate the situation. Fixation inaccuracy and predictive drift (Kowler and Steinman, 1979a, b) both influence the location of the target on the retina. These phenomena, if not corrected for, may conceivably cause a bias in the $R$ - and $\Phi$-scatter of saccade vectors. Since fixation noise is not of interest for the purpose of the present analysis, its effect was removed by a correction procedure (see below).

(2) Metrical noise. Saccade metrics can also vary because the computation of saccade amplitude and direction, by the saccadic system, is also subject to noise. In the present paper the latter type of noisy variations is investigated.

\section{Removal of fixation noise}

Prior to the analysis of the actual saccades, the ensembles of initial and final fixation positions from all test trials were aligned on the starting and final positions of the target, respectively. This was done by computing the center of gravity of initial and final fixation positions (averaged over $10 \mathrm{msec}$ ) of all successful trials (definition, see above). This procedure yielded the d.c. and gain calibration parameters of that particular session. For the largest amplitude sessions ( $R=50 \mathrm{deg}$ ), where eye positions up to an eccentricity of $25 \mathrm{deg}$ were required (see above), we have corrected for the inherent slight nonlinearity of the eye movement recording system by applying the linearization procedure, described in detail by Bour, Van Gisbergen, Bruyns and Ottes (1984).

In order to eliminate the effect of fixation noise, we have analyzed our data according to the following procedure:

Retinal error $(E)$ defined as the difference vector between target position and first-saccade onset eye position, was computed for all succesive trials. Retinal error in each trial was considered as the relevant stimulus for the saccadic system. Since we wished to compare the first-saccadic response $(S)$ of all trials even though $E$ differed from trial to trial, $S$ was normalized with respect to the stimulus step $T$ by taking $\left|S_{n}\right|=|T| \cdot(|S| /|E|)$, where $\left|S_{n}\right|$ is the amplitude of the normalized saccade vector. The direction difference between the normalized saccade vector $S_{n}$ and retinal error vector $E$ was kept the same as between the first-saccade vector $S$ and $E$.

A simple numerical example may illustrate this procedure. Suppose that a target is presented at +20 deg horizontal and that in trials 1 and 2, the onset fixation positions of the eye are +5 deg and -5 deg horizontal, respectively. This implies that the retinal error vector is $15 \mathrm{deg}$ horizontal for trial 1 and $25 \mathrm{deg}$ horizontal for trial 2. Suppose that in trial 1 the subject makes a first-saccade of $12 \mathrm{deg}$ horizontal and in trial 2 a 20 deg horizontal saccade. In both cases, the amplitude gain is 0.8 . Since the actual target eccentricity was at $20 \mathrm{deg}$, the response gains are multiplied by 20 to yield a normalized amplitude of 16 deg horizontal for both trials. Hence, despite the huge scatter in onset positions in this example, both trials yield an identical normalized saccade vector $S_{n}$.

Figure 1A shows the scatter plot of saccade endpoints in the $(R, \Phi)=(2,225)$ session (subject $R J B$ ) before application of the correction procedure. As can be seen in this figure, there is considerable scatter in the metrics of these responses and, in addition, $R$-scatter seems to be larger than $\Phi$-scatter. Part of the scatter, however, is caused by noisy presaccadic fixations of the eye. The result of cancelling out this type of noise by the procedure outlined above has been depicted in Fig. 1B. Note that the shape of the scatter plot of normalized saccade vectors now looks even more elongated along the $R$-direction that in the uncorrected plot. In the results that follow, we only show normalized saccade vector plots in the format of Fig. 1B.

In order to quantify the shape of the normalized saccade vector endpoint distribution (normalized saccade endpoints, for short), a simple measure has been defined. Histograms were constructed along an orthogonal coordinate system $(p, q)$, defined as explained in Fig. 2 . The resulting histogram along the $q$-axis was taken as the amplitude histogram of the saccades whereas the new $p$-coordinates of the endpoint data provide a measure for the direction scatter. Standard deviations, for amplitude and direction, $\sigma_{R}$ and $\sigma_{\phi}$ respectively (both in deg), taking into account all the data points, were then computed. In this paper we have adopted $\sigma_{\phi}$ as a measure of $\Phi$-scatter.

The bin width in the histograms was chosen as follows: amplitude (or direction) range ( $\rho)$ of 


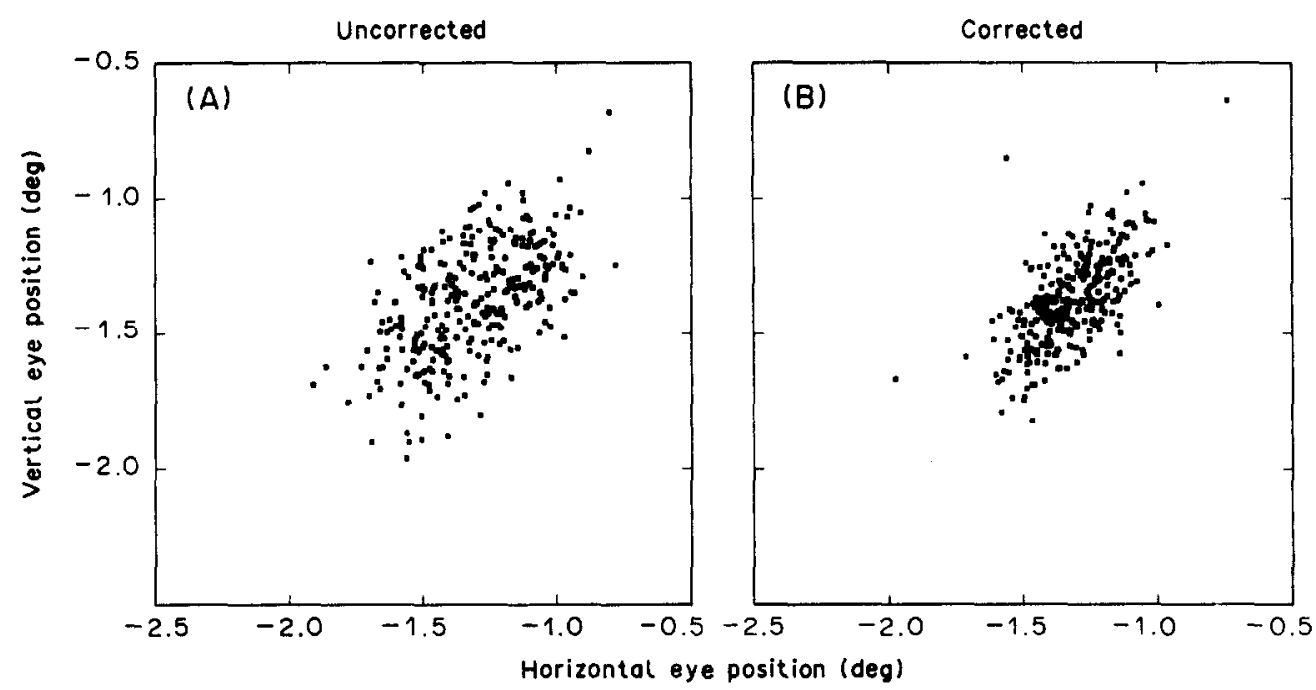

Fig. 1. Illustration of fixation-scatter removal. (A) Endpoints of uncorrected saccade vectors. Subject RJB; target position $(R, \Phi)=(2,225) \mathrm{deg}$. Number of saccades: 282 . All saccade onset positions have been shifted to the origin so that the vector endpoints partly reflect the scatter in fixation positions. Note that $R$-scatter exceeds $\Phi$-scatter. (B) Normalized saccade vector endpoints as in (A) after applying the correction procedure outlined in Methods. Endpoints now reflect only the metrical noise. Note that the

distribution of vector endpoints is even more elongated along the $R$-dimension after correction.

the saccades was taken as the difference between the largest-but-two and smallest-but-two values. The binwidth $(B W)$ of the histograms is then given by $B W=\rho / \sqrt{N}$, with $N$ the total number of successful trials.

\section{Histogram skewness}

It can be shown that the two noise sources in the ensemble coding model, (population location jitter and rate scatter), considered extensively in the Discussion, yield different predictions concerning the shape of the $R$-scatter histograms. Because the collicular motor map is highly nonhomogeneous, it can be shown that

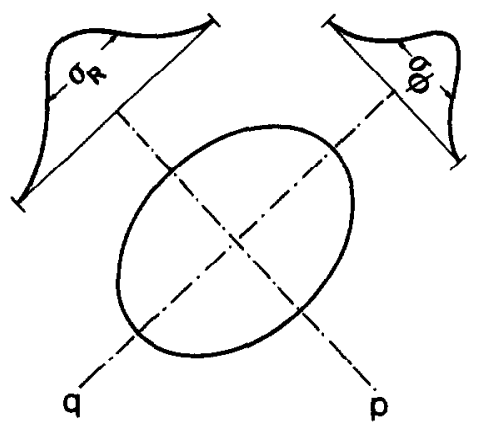

Fig. 2. Schematic illustration of the applied measures for the scatter parameters in this paper. An orthogonal coordinate system $(p, q)$, where the extension of the $q$-axis intersects the fixation spot in the below-left corner, is defined through the center of the scatter plot. Histograms along the $q$ $(R$-scatter) and $p$-axes ( $\phi$-scatter) involving all data points are constructed and standard deviations, $\sigma_{R}$ and $\sigma_{\phi}$, respectively, are computed.
Gaussian location jitter, in the model, should lead to a positively skewed distribution of saccade amplitudes and a Gaussian distribution of saccade directions (skewness $=0$ ). By contrast, as will be explained later, rate scatter in the motor map will cause the distribution of amplitudes to be the same as the assumed variation in firing rates. Thus, rate scatter also allows the possibility that the amplitude distribution can become negatively skewed. A problem with the skewness measure is that it is very sensitive to noise (see also Van Opstal \& Van Gisbergen, $1987 b$ ) so that it is not very useful to compute its value directly from the raw data. We have therefore smoothed the histograms by fitting them with a 6th-order polynominal function and have computed skewness from the best-fit function (see Van Opstal \& Van Gisbergen, $1987 \mathrm{~b}$, for more details). The skewness values of the amplitude scatter histograms are given in Table 2 of the Discussion section.

\section{RESULTS}

Normalized saccade endpoint scatter plots for different target eccentricities

In Fig. 3 we have plotted normalized saccade endpoints for six different target eccentricities of subject RJB ( $R$-sequence), all on the same scale. Notice that in all cases scatter along the $R$-direction is larger than along the orthogonal direction. This appears to be a consistent finding 

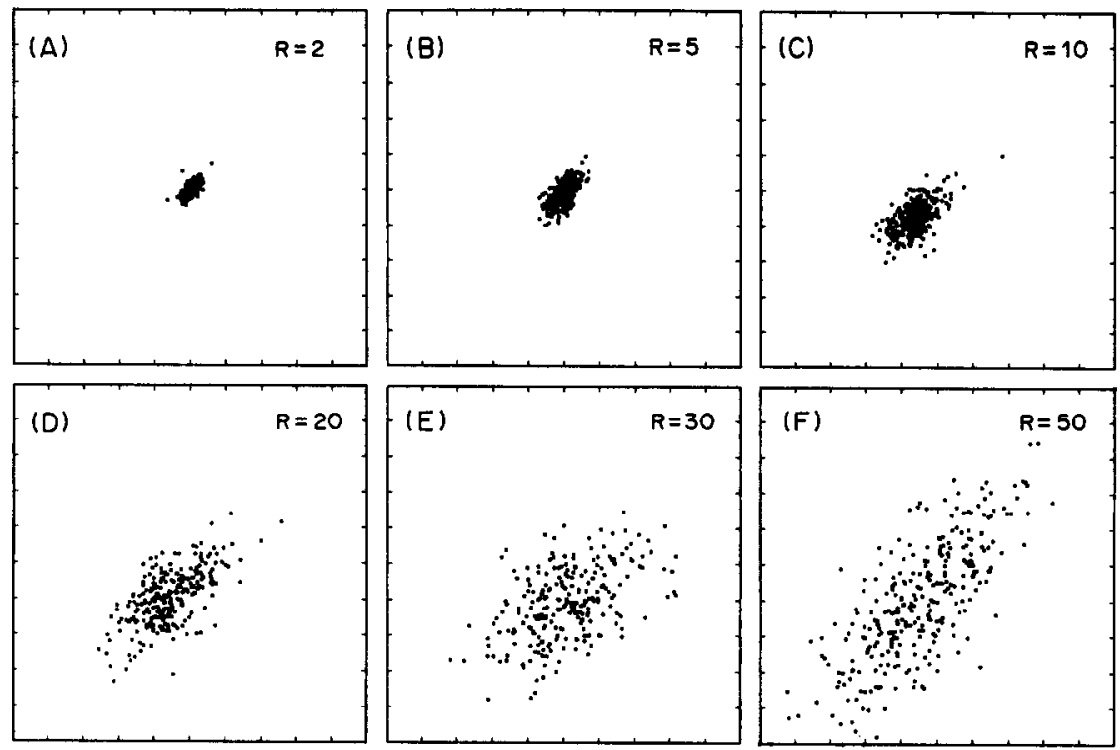

Fig. 3. Normalized saccade endpoint scatter plots for six different target eccentricities in the $R$-series experiments. Both axes and all panels are on the same scale (one degree per division). Target eccentricity (in [deg]) is indicated in each box ( $\Phi=225 \mathrm{deg}$ in all cases). Note that for all target positions $R$-scatter exceeds $\Phi$-scatter. Note also that absolute amount of scatter increases with amplitude. Data from subject RJB.

in all subjects for all target positions presented (see also Figs 5 and 6). Notice also that the amount of scatter increases strongly with amplitude (see below and Discussion).

\section{Histograms of amplitude and direction scatter}

In order to get a quantitative grasp on data such as in Fig. 3, we have constructed histograms of first-saccade amplitudes and directions for each target position (see Methods). Three typical examples of such histogram pairs (subject RJB; $R=5,20$ and $40 \mathrm{deg} ; \Phi=225$ deg), shown in Fig. 4, confirms that there is indeed more scatter in amplitude than along the orthogonal direction dimension. Since these histograms were always single-peaked, it makes sense to characterize their width and their shape by two sets of parameters, i.e. standard deviation ( $\sigma_{R}$ for amplitudes and $\sigma_{\Phi}$, for directions) and skewness $\left(S_{R}\right.$ and $S_{\phi}$, respectively, see Methods and Fig. 2).

\section{Relation between scatter parameters and retinal target position}

Figure 5A summarizes the dependence of the scatter parameters, $\sigma_{R}$ (solid symbols) and $\sigma_{\Phi}$ (open symbols) on target eccentricity for all four subjects. It can be seen that both kinds of scatter show a roughly linear increase with eccentricity. Figure 5B shows that the same parameters have no obvious relation with target direction. In
Fig. $5 \mathrm{C}$ the amplitude scatter parameter $\sigma_{R}$ has been plotted as a function of the direction scatter parameter $\sigma_{\Phi}$ for all subjects and target positions. These data can be approximated by a straight line with a slope clearly above 1.0, which confirms that $\sigma_{R}$ is consistently larger than $\sigma_{\phi}$ (see Figs 1 and 3).

Figure 6A shows that there is no significant relation between target eccentricity and the ratio $\sigma_{R} / \sigma_{\phi}$. This result, showing that the degree of ellipticity in normalized saccade endpoint scatter plots of the type shown in Figs $1 B$ and 3 was independent of amplitude, was also obtained in the other subjects. Figure 6B demonstrates that the degree of ellipticity of these plots has no obvious relation with target direction. In all cases the ratio $\sigma_{R} / \sigma_{\Phi}$ exceeds 1.0 , which would be the value predicted by the combination of location jitter and an isotropic motor map (see Discussion). Table 1 summarizes the mean values of the scatter ratio for our four subjects, computed from the $R$-series experiments, together with their standard deviations.

Taken together, the data yield a fairly constant value for $\sigma_{R} / \sigma_{\phi}$ which is in line with the location scatter version of the ensemble coding model (see below).

\section{Test on presence of long-term trends}

In order to check the extent to which the saccadic responses were affected by long term 


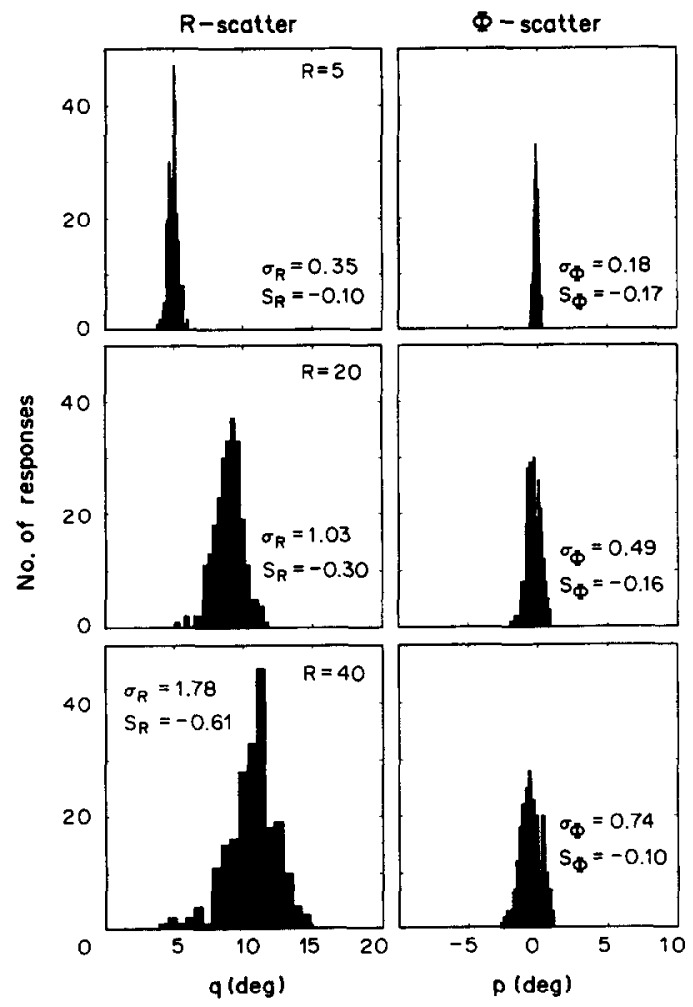

Fig. 4. Histograms of $R$-scatter (left-hand column) and $\Phi$-scatter (right-hand column). Target positions: $R=5$ (top row), 20 (middle row) and $40 \mathrm{deg}$ (bottom row). $\Phi=225 \mathrm{deg}$ in all cases. Subject RJB. All abcissas are on the same relative scale ( $5 \mathrm{deg}$ per division) in which the zero offset position has been shifted for illustrative purposes. Ordinates: number of saccades. Scatter parameters of width $\left(\sigma_{R}\right.$ and $\sigma_{\phi}$, in [deg]) and skewness $\left(S_{R}\right.$ and and $S_{\phi}$, [dimensionless]) of the histograms are indicated in each box. Note that $R$-scatter histograms are slightly negatively skewed and that, in all cases, the $R$-scatter histogram is broader than the $\Phi$-scatter histogram. effects which might be caused by fatigue or the gradual emergence of predictive tracking, we have plotted saccade gain, the normalized magnitude of the vectorial retinal error remaining after the first saccade, mean first saccade velocity (see Methods for definition) and first saccade latency as a function of trial number. As can be seen from the four plots in Fig. 7A-D, for a typical experimental session $(R=5 \mathrm{deg}$, $\Phi=225 \mathrm{deg}$; subject RJB), no obvious dependence of these saccade parameters on trial number could be found. In general, normalized saccade gain, remaining retinal error, and onset latency had no significant relation with time in $75 \%$ of the experimental sessions.

We have also checked whether the amount of variation of these parameters showed a significant trend with time by computing their standard deviations in subsequent blocks of 15 trials. In all subjects tested it was found that the standard deviation in these parameters did not vary significantly with time in the large majority (more than $90 \%$ ) of sessions (not shown).

In a substantial number $(60 \%)$ of experiments we found that mean eye velocity showed a slight but systematic negative trend with time. In the large majority of sessions, however, the decrease in mean velocity did not exceed $10 \%$. As will be explained in the Discussion, it is important to know whether metrical and dynamical parameters are somehow correlated with each other. It is not a trivial matter to investigate this because saccade amplitude and (mean) eye velocity are related through the so-called main
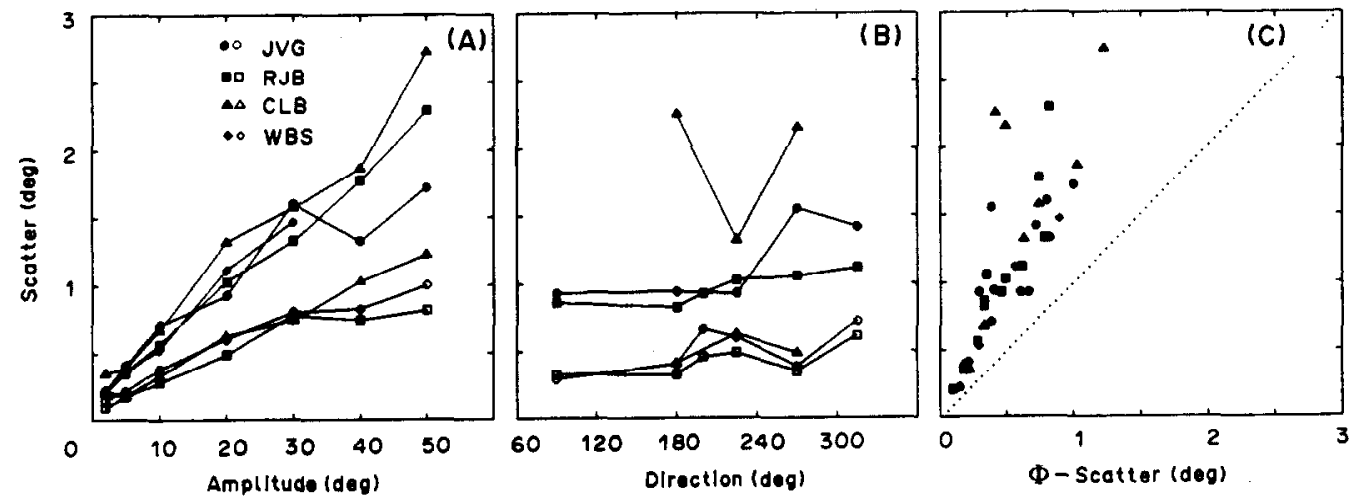

Fig. 5. Relation between scatter parameters $\left(\sigma_{R}\right.$ and $\left.\sigma_{\phi}\right)$ and target position for all subjects. Subjects are indicated by different symbols (JVG: circles; RJB: squares; CLB: triangle and WBS: diamonds). (A) $\sigma_{R}$ (solid symbols) and $\sigma_{\phi}$ (open symbols) as a function of target eccentricity. Note that both parameters increase approximately linearly with eccentricity and that $\sigma_{\phi}$ is always smaller than $\sigma_{R}$. Data from $R$-series experiments. (B) The same parameters as in (A) as a function of target direction. Note that the amount of scatter is independent of direction. For two directions in subject CLB ( $\Phi=180$ and $270 \mathrm{deg}$ ) an unexplained excessive amount of $R$-scatter was observed. Data from $\Phi$-series experiments. (C) $\sigma_{R}$ and $\sigma_{\phi}$ plotted against each other. Note that the data can be described approximately by a straight line whose slope clearly exceeds 1.0 (dashed line). 

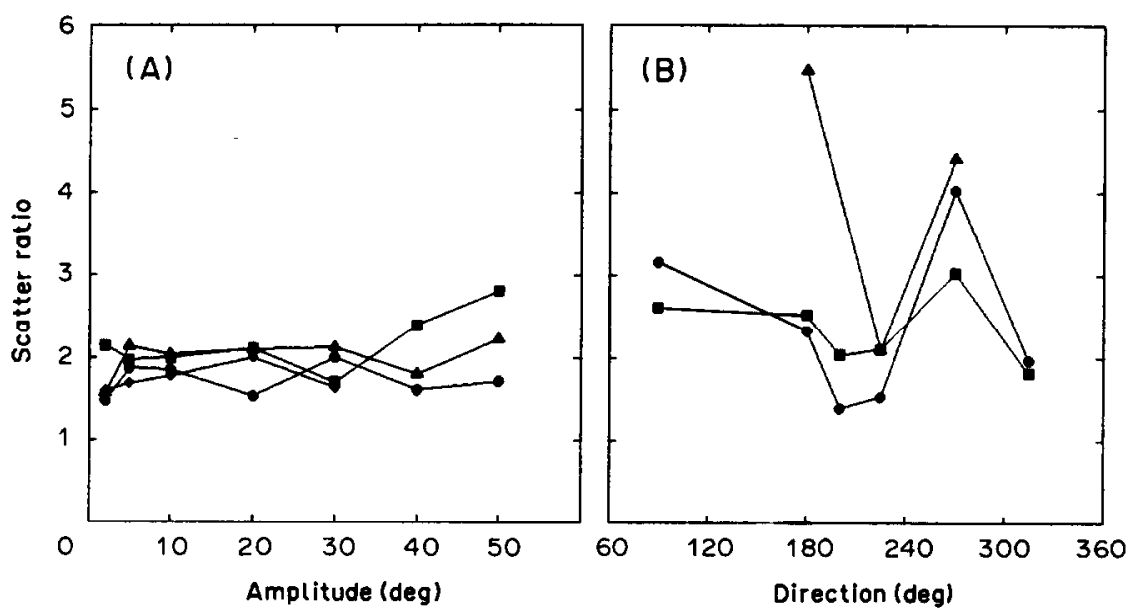

Fig. 6. Scatter ratio $\left(\sigma_{R} / \sigma_{\phi}\right)$ as a function of target position for all subjects. Same symbols as in Fig. 5A. (A) Scatter ratio as a function of target eccentricity. Note that in all subjects this ratio is independent of eccentricity [correlation coefficient for RJB: $r=0.63(n=7$, NS); JVG; $r=0.05 \quad(n=7$, NS); CLB: $r=0.37(n=7$, NS); WBS: $r=0.25(n=5$, NS)]. (B) Scatter ratio as a function of target direction. Note that in all except two cases (subject CLB: $\Phi=180$ and $270 \mathrm{deg}$ ) the scatter ratio is roughly comparable with the mean value found in the $R$-series (see A).

sequence (Bahill, Clark \& Stark, 1975). In order to circumvent effects of this inherent relationship in our analysis, we have computed the standard deviations, from blocks of 15 trials, separately for gain and mean eye velocity, and have determined their correlation coefficient. If variations in arousal would somehow be a major underlying factor in causing the characteristic appearance of normalized saccade endpoint plots (Figs 1B and 3), one would expect a clear covariation between these two parameters. In the large majority (34/38) of experimental sessions, however, no significant correlation could be found (see Table 4 and Discussion).

Thus, the general picture emerging from these analyses is that the normalized saccade endpoint distributions were not affected by learning effects nor by some kind of fatigue (see e.g. Schmidt, Abel, Dell'Osso \& Daroff, 1979) or changes in alertness during the experiment. Therefore it is unlikely that the observed scatter in these responses is due to such factors (see also Discussion). The absence of long term trends in the metrical responses does not guarantee, of course, that the behaviour of the subjects was

Table 1. Mean scatter ratio, a measure for the amount of anisotropy in the collicular motor map (see Discussion and Appendix) for all subjects

\begin{tabular}{lll}
\hline Subject & $\sigma_{R} / \sigma_{\diamond}$ & SD \\
\hline JVG & 1.73 & 0.19 \\
RJB & 2.16 & 0.35 \\
CLB & 2.00 & 0.23 \\
WBS & 1.74 & 0.14 \\
\hline
\end{tabular}

completely stationary. As will be explained in the Discussion, the nonstationarity observed in certain scatter parameters can be useful in discriminating between the two hypothetical noise sources in the extended ensemble coding model (see Discussion).

\section{DISCUSSION}

\section{General remarks}

In this paper we have confirmed and extended the earlier finding that normalized saccade endpoint scatter is more elongated along the eccentricity dimension than along the direction dimension (Deubel, 1987). By using our fixation-noise removal procedure, we have shown in humans subjects that his phenomenon cannot be due to systematic inaccuracies of presaccadic fixations; elimination of fixation scatter may even enhance it (Fig. 1). We have also checked whether slow changes in performance could have contributed to this phenomenon but could not find any consistent trend with time, neither for the parameters saccade gain, normalized residual retinal error and latency nor for the variation in these parameters (Fig. 7).

In addition to Deubel's (1987) observations we have found that the scatter width parameters both along the eccentricity as well as along the direction dimension $\left(\sigma_{R}\right.$ and $\sigma_{\phi}$, respectively) increase with target eccentricity but are roughly constant for equal-eccentricity target positions in different directions. 

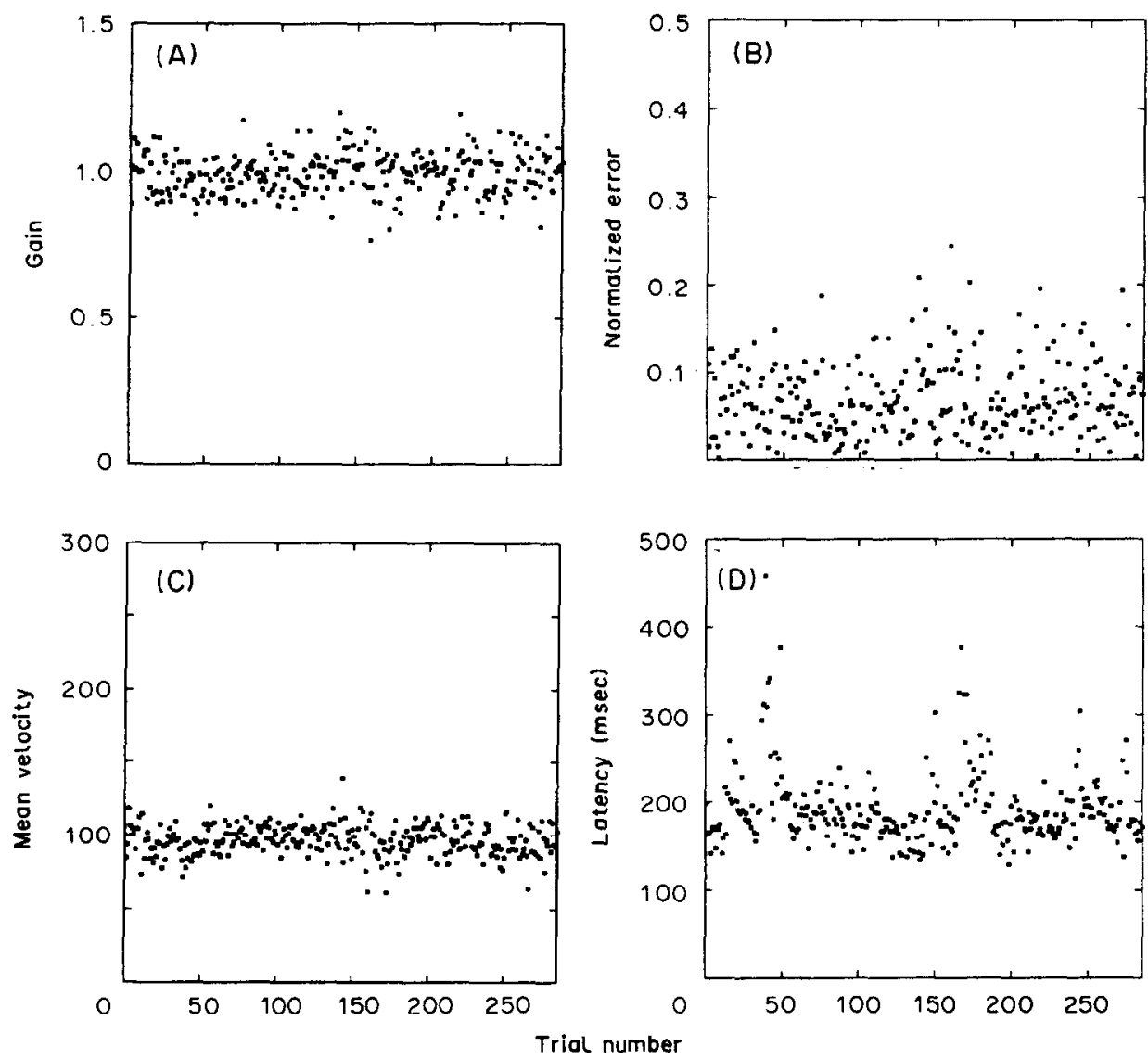

Fig. 7. Absence of long-term trends. (A) Saccade gain does not show a significant relation with trial number (correlation coefficient, $r=0.09, n=292$, NS). The standard deviation in saccade gain (not shown), computed in subsequent blocks of 15 trials, is also independent of block number $(r=0.27$; NS; number of blocks, $n=17)$. Subject RJB, target position $(R, \Phi)=(5,225)$ deg. Number of responses: 292 . (B) Normalized magnitude of retinal error remaining after the first saccade of each trial as a function of trial number $(r=0.08$; NS). Correlation of the standard deviation with block number is $r=0.22$ (NS). (C) Mean velocity (amplitude/duration, in $\mathrm{deg} / \mathrm{sec}$ ) as a function of trial number $(r=-0.04$; NS). Standard deviation: $r=0.20$ (NS). (D) Onset latency plotted against trial number ( $r=0.09$; NS). Standard deviation, $r=-0.21$ (NS).

The ratio of $\sigma_{R}$ and $\sigma_{\phi}$, a measure for scatter plot ellipticity (see Fig. 2), was roughly constant and always larger than 1.0 for all target eccentricities and directions studied in all four subjects (Figs $5 \mathrm{C}$ and 6; see also below).

Can noisy variations in the collicular motor map account for saccade scatter?

Properties of the motor map. Three features of the collicular motor map are worth noticing: first, as an approximation, the collicular motor map is organized in polar coordinates $(R, \Phi$; Robinson, 1972) in the sense that saccade amplitude $(R)$ and direction $(\Phi)$ are represented along roughly orthogonal dimensions. Second, the map is nonhomogeneous in that a disproportionately large area of the $S C$ map is dedicated to the generation of small saccades (Robinson, 1972; Sparks, 1986). Third, on the basis of
Robinson's (1972) electrical stimulation data, Ottes et al. (1986) concluded that the motor map is slightly anisotropic since it is more expanded along the $\Phi$-representation than along the $R$ representation.

We wondered whether these three properties of the motor map could underly the observed elliptical scatter of normalized saccade endpoints in monkeys and humans (Deubel, 1987; this study) by assuming noisy variations at the level of the collicular motor map. This possibility will be discussed further below by considering the effect of two hypothetical noise sources in the motor map within the frame work of the ensemble coding model (Van Gisbergen et al., 1987).

Brief recapitulation of the ensemble coding model. In the model, which has been extensively described elsewhere (Ottes et al., 1986; Van 
Gisbergen et al., 1987; Van Opstal \& Van Gisbergen, 1989), both saccade amplitude and direction are specified by the location of a large population of active cells in the deeper layers of the superior colliculus. The location of the active population is determined by a so-called afferent mapping function, which describes the correspondence between retinal coordinates $[(R, \Phi)$, in deg] and $\mathrm{SC}$ coordinates [(u,v) in $\mathrm{mm}]$. In line with the complex-logarithmic nature of this mapping function, the resulting collicular map can be conceived of as a representation of the saccade vector in polar coordinates, where the collicular $u$-axis (see Fig. 8) mainly represents saccade amplitude $(R)$ and the orthogonal $v$-axis predominantly determines saccade direction $(\Phi)$ (see below). The model illustrates how the location of the recruited population can code the appropriate saccade metrics by assuming that each recruited cell contributes to the total saccade by generating a small movement contribution (efferent mapping stage) in a direction which corresponds to its associated retinal locus. The amplitude of this contribution is determined by the mean firing rate of the cell in combination with its synaptic connection strengths with the premotor system downstream (see Van Gisbergen et al., 1987 and Van Opstal \& Van Gisbergen, 1989, for more details).

In the original ensemble-coding model no attempt had been made to account for the variability in saccade metrics. In this paper we extend the model by considering two possible sources of noise in the collicular motor map: location jitter and rate noise (Fig. 8). First, we assume that the location of the center of the active population has a Gaussian distribution (standard deviation: $\sigma_{s}$, see Fig. 8B) around the mean location specified by the afferent mapping stage (location jitter). Second, we separately wish to consider the possibility that the mean firing rate of all recruited cells in the population is subjected to noise (Fig. 8C), so that there is trial-totrial variability in the total amount of spikes in the population activity profile (rate noise).

Model predictions on the effects of location jitter. Since the motor map is nonhomogeneous, the model predicts that absolute $R$ - and $\Phi$-scatter of the resulting normalized saccade endpoints increase roughly linearly with target eccentricity when the amount of population activity jitter from trial-to-trial is identical for all locations in the motor map (translation invariance). It should be realized that in the extreme case of a homogeneous motor map this
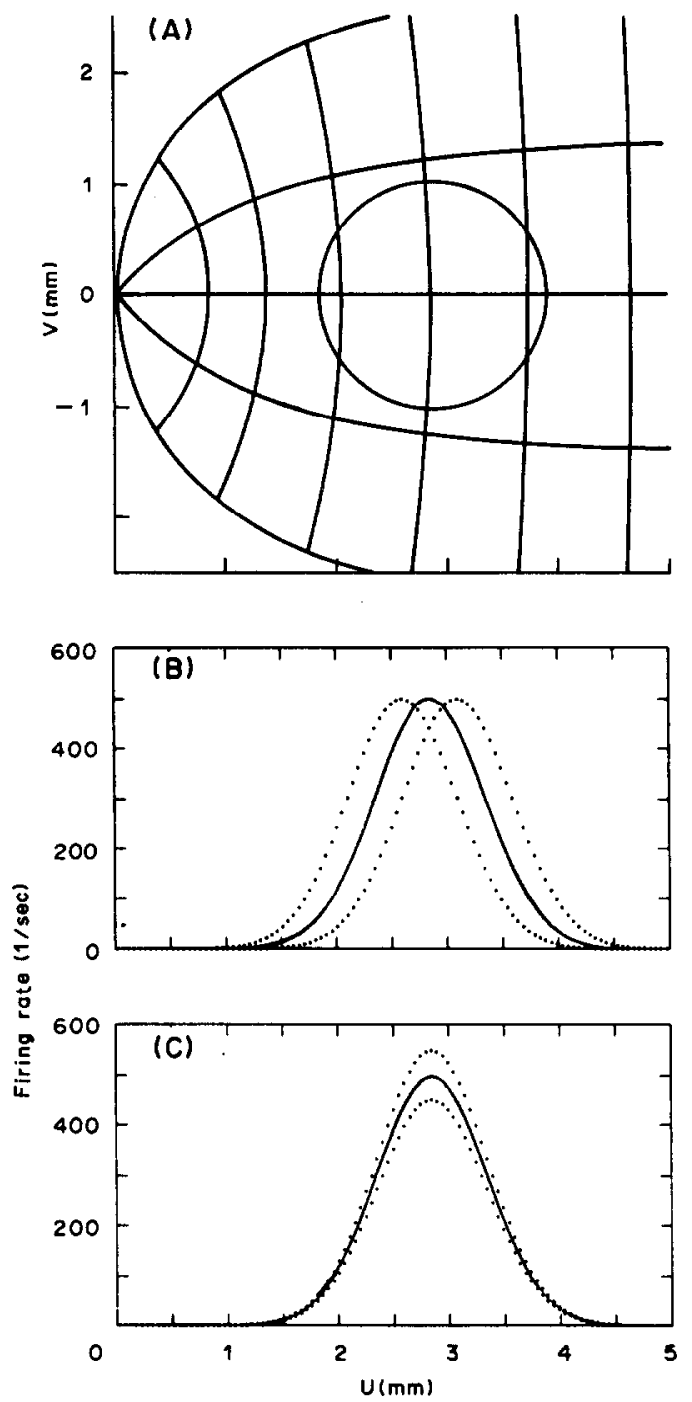

Fig. 8. Extended version of the ensemble coding model with the two different noise sources described in the Discussion. (A) View on the collicular surface with population activity profile, for an $R=20, \Phi=0$ deg saccade, centered at $u=2.85 \mathrm{~mm}$ on the horizontal meridian representation Circle denotes $2 \sigma(=1 \mathrm{~mm})$ activity border. (B) Location jitter: cross-section through the population activity profile along the horizontal meridan representation $(v=0 \mathrm{~mm})$ showing the effect of location jitter on the center location of the population activity. The location noise is assumed to be Gaussian, rotation-symmetrical and translation invariant throughout the motor colliculus for different saccades. The resulting saccade vectors wil be distributed elliptically. (C) Firing rate-scatter: Scatter in firing rates is assumed to affect all neurons of the population simultaneously. Therefore the total total number of spikes generated by the population is subject to noise which will result in scatter of saccade amplitudes.

model yields a constant amount of scatter for all target eccentricities. Therefore, the property of map nonhomogeneity is essential to explain our data (Figs 3 and 5a) in terms of the population jitter model. 
In addition, the model predicts that the ratio of $R$-scatter and $\Phi$-scatter is approximately constant for target positions throughout the visual field apart from very small amplitude saccades, where this ratio is expected to be larger (see Appendix). This ratio, in the pure location-jitter model, depends entirely on the degree of anisotropy of the motor map: if the map is isotropic, $R$ - and $\Phi$-scatter will be equal. In the case of map anisotropy of the type found in the monkey (Ottes et al., 1986), however, $R$-scatter will exceed $\Phi$-scatter. For a more detailed analysis of the consequences of the pure location-jitter model the reader is referred to the Appendix.

It is possible to get an impression of the amount of location jitter which must be assumed to explain our findings and to test the translation-invariance assumption. If the model is correct, inverse mapping of the endpoint scatter onto collicular coordinates, should yield a Gaussian distribution of reconstructed population activity centers which is invariant for different target positions. Furthermore, due to the nonhomogeneous nature of the motor map, the model predicts that the distribution of saccade amplitudes should be positively skewed, whereas saccade directions are expected to be distributed in a Gaussian fashion. Finally, in the case of nonstationary jitter it is expected that variations in saccade amplitude and saccade direction will covary (see below).

Model predictions on the effects of rate noise. In this scenario only the amplitude of saccades will be noisy since firing rate in the model only determines the amplitude of each cell's contribution (see above). The expected amplitude distribution in this case reflects the distribution of noise in firing rates because, in the model, population firing rate and saccade amplitude are linearly related (Van Gisbergen et al., 1987). Since firing rate in the ensemble coding model is a multiplicative variable (Van Gisbergen et al., 1987, for details), rate noise in the model has a multiplicative effect. Therefore this model, as the location-jitter model, yields a proportional increase of amplitude scatter with eccentricity. According to this scheme, the shape of the saccade amplitude histograms (as expressed by the skewness parameter) directly reflects the underlying scatter in population firing rates. Saccade directions, however, will not vary if rate-scatter is the only noise source, because, in the model, saccade direction is not determined by firing rates of collicular neurons as such but by their relative connection strengths with the horizontal and vertical premotor system, which depend exclusively on their location within the motor map (see above). The degree of ellipticity in normalized saccade endpoint plots therefore reduces to the extreme case of elongated radial lines.

\section{Test of the two models}

Experimental findings in the present study. As can be seen in Figs 3 and 5, the experimental data can be described succinctly by a linear increase of the two scatter parameters ( $\sigma_{R}$ and $\sigma_{\phi}$ ) with target eccentricity. The linear increase in absolute amplitude scatter with target eccentricity is in line with the predictions of both the pure-location jitter model and the rate noise scheme. Also the finding that this scatter parameter is independent of target direction is supported by both models. The result that direction scatter also increases linearly with target eccentricity is consistent with the jitter model, but cannot be explained by rate noise alone. Therefore, the observation that the scatter ratio is roughly independent of both target eccentricity (for $R$ sufficiently large, see Appendix) and target direction (Fig. 6) can, at least in principle, be understood from the pure location-jitter scheme if it is assumed that this ratio reflects the amount in anisotropy of the collicular motor map. However, for small amplitudes it is expected from this model that the scatter ratio should be about twice as large (see Appendix). This prediction is not supported by the data of Fig. 6.

To illustrate the implications of the pure population jitter model more directly, the scatter data of subject RJB have been replotted onto a collicular motor map (Fig. 9), whose amount of anisotropy was taken as 2.16 (Table 1). We have computed that the reconstructed amount of population activity jitter in the collicular motor map is about $60 \mu \mathrm{m}$ on the average (not shown) which is small $(12 \%)$ in comparison with the width of the population activity profile itself ( $\sigma=0.5 \mathrm{~mm}$, Ottes et al., 1986). Also it should be noted that, in line with the model, the scatter histograms in the colliculus for the different target positions, are reasonably comparable and almost circular-symmetrical (mean ratio $\left.\sigma_{R} / \sigma_{\Phi}=1.0 ; \mathrm{SD}=0.2\right)$. The largest deviations from rational symmetry were found for small amplitudes $(R=2$ and $R=5 \mathrm{deg}$; see also Appendix). 


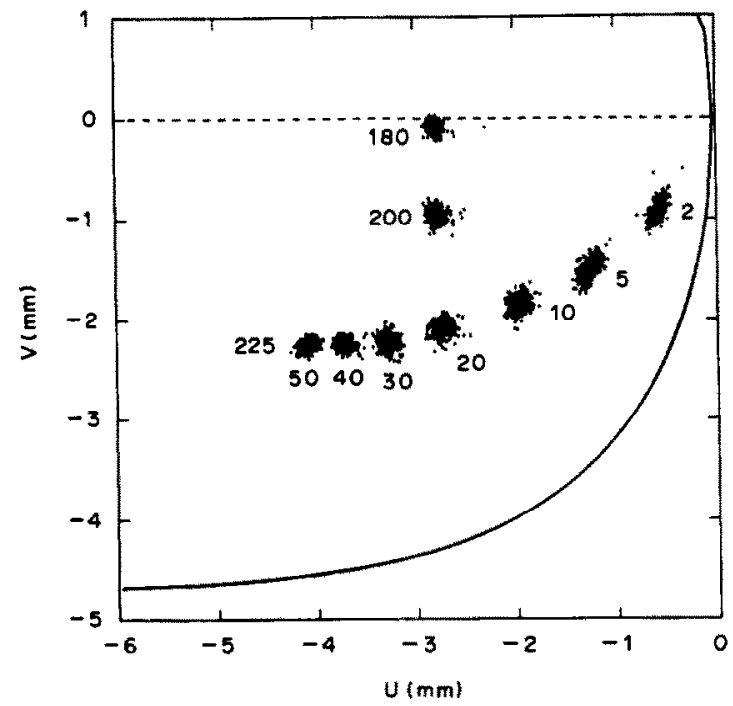

Fig. 9. Data of subject RJB replotted in collicular coordinates. The inverse efferent mapping function of the colliculus, used in the remapping procedure (see Appendix in Ottes et al., 1986) had the parameters $B_{u}=1.4 \mathrm{~mm}$; $B_{r}=3.0 \mathrm{~mm} / \mathrm{rad} ; A=3.0 \mathrm{deg}$. See Appendix for further explanation.

The implication of the location jitter model, that skewness of the amplitude histograms should be positive in all cases is not supported by the experimental data. As can be seen in Table 2 , most sessions actually (27/38) yielded negative skewness values. This finding is compatible with the rate-scatter model if it is assumed that normally firing rates are close to some maximum value, so that variations can manifest themselves only in lower rates and hence in a decrease of saccade amplitude. It should be noted, however, that the phenomenon of negative skewness is rather subtle and, in most cases, not immediately clear from visual inspection of the data (see Fig. 4).

It should be stressed, at this point, that the two models yield different predictions for the degree of covariation between the amplitude and direction variability of saccades. Since in the rate noise model noise causes variability in saccade amplitude but not in saccade direction there is no reason to expect covariation. By contrast, the location jitter model predicts covariation between saccade amplitude and direction variations when the amount of population jitter is nonstationary. In order to check the extent to which saccade amplitude and direction actually covaried in a given experimental session, we computed the standard deviations of amplitude and direction separately in blocks of 15 trials. The complete set of correlation coefficients for all subjects and target positions is given in Table 3. It is clear from these data that in all cases except one these two parameters do not have a significant correlation. This result strongly supports a scheme with independent control of amplitude and direction, such as the rate noise version of our model (see also Becker \& Jürgens, 1979), and cannot be explained by the pure location-jitter version. Since the extreme versions of the model can explain some

Table 2. Skewness values of the amplitude scatter histograms (see Methods) for all experimental sessions

\begin{tabular}{|c|c|c|c|c|c|c|c|c|}
\hline \multicolumn{5}{|c|}{$R$-series experiments, $\Phi=225$} & \multicolumn{4}{|c|}{$\Phi$-series experiments, $R=20$} \\
\hline$R$ & JVG & RJB & CLB & WBS & $\Phi$ & JVG & RJB & CLB \\
\hline $\begin{array}{r}2 \\
5 \\
10 \\
20 \\
30 \\
40 \\
50\end{array}$ & $\begin{array}{r}0.21 \\
-0.23 \\
-0.81 \\
0.21 \\
0.08 \\
-0.42 \\
-0.20\end{array}$ & $\begin{array}{l}-0.63 \\
-0.10 \\
-0.40 \\
-0.30 \\
-0.27 \\
-0.61 \\
-0.99\end{array}$ & $\begin{array}{r}0.72 \\
-0.31 \\
-0.64 \\
-0.94 \\
-0.17 \\
-0.75 \\
-0.47\end{array}$ & $\begin{array}{r}0.75 \\
-0.40 \\
0.00 \\
-0.15 \\
-0.29\end{array}$ & $\begin{array}{r}90 \\
180 \\
200 \\
270 \\
315\end{array}$ & $\begin{array}{r}-0.47 \\
-0.09 \\
-0.13 \\
0.05 \\
-0.52\end{array}$ & $\begin{array}{l}-0.61 \\
-0.47 \\
-0.94 \\
-0.37 \\
-0.50\end{array}$ & $\begin{array}{r}-0.34 \\
0.05\end{array}$ \\
\hline
\end{tabular}

Table 3. Correlation coefficients between the standard deviations, computed in blocks of 15 trials, of saccade amplitudes and directions for all sessions. Only one case (a) yielded a significant correlation $(P<0.01)$

\begin{tabular}{|c|c|c|c|c|c|c|c|c|}
\hline \multicolumn{5}{|c|}{$R$-series experiments, $\Phi=225$} & \multicolumn{4}{|c|}{$\Phi$-series experiments, $R=20$} \\
\hline$R$ & JVG & RJB & CLB & WBS & $\Phi$ & JVG & R.JB & CLB \\
\hline 2 & 0.02 & 0.05 & 0.28 & 0.52 & 90 & -0.36 & 0.23 & \\
\hline 5 & -0.07 & 0.45 & 0.06 & -0.39 & 180 & 0.12 & 0.38 & 0.07 \\
\hline 10 & 0.40 & 0.25 & -0.16 & 0.27 & 200 & -0.15 & 0.12 & \\
\hline 20 & 0.03 & 0.44 & 0.15 & -0.23 & 270 & 0.44 & 0.39 & 0.15 \\
\hline 30 & $0.64^{a}$ & 0.12 & 0.23 & 0.20 & 315 & -0.22 & 0.17 & \\
\hline 40 & 0.05 & 0.40 & 0.27 & & & & & \\
\hline 50 & -0.35 & 0.27 & 0.13 & & & & & \\
\hline
\end{tabular}


but not all features of the data, a model where both types of noise do contribute to saccade endpoint scatter seems more realistic.

Evidence from electrical stimulation studies. Electrical stimulation studies in the deeper layers of the monkey superior colliculus, currently performed in our laboratory (manuscript submitted), reveal that lowering the electrical stimulation strength strongly influences the amplitude of the forthcoming saccades ( $c f$. Sparks \& Mays, 1983) but not their direction. It is also a consistent finding that repeated stimulation with a constant current strength yields elliptical saccade endpoint histograms just as we found in the present behavioural experiments. These findings can be explained by the ensemble coding hypothesis by assuming that lowering electrical current intensity affects the size of the activated population of movement cells, either by an overall decrease of the mean firing rate within the population, or by shrinkage of the population profile. In either case, the decrease in the total number of spikes in the activity profile with decreasing stimulus intensity could account for the reduction in saccade amplitude.

The fact that saccade endpoints are distributed elliptically at constant current stimulation conditions provides strong support for the assumption that the endpoint distributions are the result of noisy processing at the motor stage rather than in the visual afferent pathways, but does not prove that they occur at the level of the collicular motor map (see below).

Recently, Lee, Rohrer and Sparks (1988) have shown that reversible inactivation of a small area in the motor colliculus may still result in normometric saccades. To explain this finding they favour a more complex ensemble coding scheme where the contribution of each cell depends also on the total population activity. Their idea, which still needs to be worked out in the explicit form of a quantitative model that allows simulations, cannot readily explain why saccade amplitude depends on electrical-current strength (Sparks \& Mays, 1983).

\section{Alternative models}

The fact that saccade vector scatter plots have elliptical shapes with axes of symmetry along the eccentricity and directional dimensions can be understood if one assumes that the subsystem which determines saccade metrics is organized in a polar-coordinate system. Theoretically, however, the ellipses may also result from a common noise source (such as arousal variations) in otherwise independent horizontal and vertical channels downstream of the motor colliculus. One may wonder whether arousal may be a factor which causes the saccade vector endpoints to be distributed elliptically in such a "Cartesian" scheme (see e.g. Van Gisbergen et al., 1985).

Trial-to-trial variations in arousal should be reflected in eye velocity fluctuations (Schmidt et al., 1979; Jürgens, Becker \& Kornhuber, 1981) and one may ask whether such fluctuations may also underly the clliptical scatter obscrved in saccade metrics. The problem of a possible relation between dynamical and metrical fluctuations has been studied for horizontal saccades by Jürgens et al. (1981). They found that after an injection of Diazepam the dynamical properties of the saccades were highly variable, but saccade gain was hardly affected. Their result suggests independent control of saccade metrics and saccade dynamics and taken together with the findings in our study that variations in mean eye velocity and saccade gain do not show a significant positive correlation in the large majority of the experiments (Table 4), makes it quite unlikely that the metrical variations in our study were caused by a fluctuating state of alertness of the subject.

In line with this interpretation, current hypotheses on the premotor saccade generator downstream of the motor colliculus also

Table 4. Correlation coefficients between the standard deviations, computed in 15-trial blocks, of saccade gain and mean eye velocity. In only $4 / 38$ sessions (a) a significant correlation $(P<0.01)$ is found

\begin{tabular}{|c|c|c|c|c|c|c|c|c|}
\hline \multicolumn{5}{|c|}{$R$-series experiments, $\Phi=225$} & \multicolumn{4}{|c|}{$\Phi$-series experiments, $R=20$} \\
\hline$R$ & JVG & RJB & CLB & WBS & $\Phi$ & JVG & RJB & CLB \\
\hline 2 & -0.06 & 0.28 & 0.12 & 0.08 & 90 & 0.17 & -0.01 & \\
\hline 5 & $0.70^{\mathrm{a}}$ & 0.45 & $0.61^{\mathrm{a}}$ & -0.13 & 180 & -0.11 & $0.74^{a}$ & 0.16 \\
\hline 10 & 0.35 & 0.18 & 0.38 & 0.41 & 200 & -0.09 & 0.09 & \\
\hline 20 & 0.10 & 0.25 & $0.81^{\mathrm{a}}$ & -0.07 & 270 & -0.26 & 0.44 & 0.03 \\
\hline 30 & -0.03 & -0.25 & 0.04 & -0.12 & 315 & 0.24 & 0.43 & \\
\hline 40 & 0.19 & -0.26 & 0.25 & & & & & \\
\hline 50 & 0.08 & -0.20 & -0.29 & & & & & \\
\hline
\end{tabular}


propose that when the pulse generator fluctuates, the hypothetical internal feedback loop of the saccadic system (see e.g. Robinson, 1975; Jürgens et al., 1981; Van Gisbergen et al., 1981) will always bring the eye on target so that the metrics of saccades would not be expected to be influenced by arousal effects. Thus, these models clearly allow for the possibility of independent scatter in metrical and dynamical parameters.

The finding of Lee et al. (1988) that local inactivation of the motor colliculus may still result in normometric saccades even though the velocity of these saccades was clearly reduced, shows once more that the metrical and dynamical properties of saccades may vary independently of one another.

An alternative conceptual model, proposed by Deubel (1987), assumes that the metrical variations are the result of the organization of the saccadic system downstream of the motor colliculus but before the decomposition stage yielding muscle-related control singles. Deubel suggested that this system is organized in a large number of directional channels, each with independent gain control. Such a model is in fact a (coarse) polar coordinate model and can explain, at least in principle, that amplitude and direction of saccades are treated as independent variables and, in addition, that the scatter of saccade vector endpoints is elliptical.

\section{Conclusions}

We have documented noisy variations in the amplitude and direction of saccades and have argued, on theoretical grounds and on the basis of data from the literature, that the noise source may be located at the level of the collicular motor map, or downstream, but before the decomposition into muscle-related control signals. The ability of two distinct noise sources in the collicular motor map to explain various features of the data is discussed on the basis of a quantitative model. We conclude that a combination of these factors would have to be assumed to explain most of the experimental phenomena. Certain alternative models cannot be excluded.

Acknowledgements-This study was supported by the Netherlands Organization for the Advancement of Pure Research (NWO) and the Brain project of the EC. The authors thank naive subjects Rob Berg, Carlo Buise and Willem Sanders for staying wide awake during the experiments.

\section{REFERENCES}

Bahill, A. T., Clark, M. R. \& Stark, L. E. (1975). The main sequence, a tool for studying human eye movements. Mathematical Biosciences, 24, 191-204.

Becker, W. \& Jürgens, R. (1979). A analysis of the saccadic system by means of double step stimuli. Vision Research, 19, 967-983.

Bour, J. L., Van Gisbergen, J. A. M., Bruyns, J. \& Ottes, F. P. (1984). The double magnetic induction method for measuring eye movement: Results in monkey and man. IEEE Transactions of the BME, 31, 419-427.

Collewijn, H., Van der Mark, F. \& Jansen, T. J. (1975). Precise recording of human eye movements. Vision Research, 15, 447-450.

Deubel, H. (1987). Adaptivity of gain and direction in oblique saccades. In O'Regan, J. K. \& Lévy-Schoen, A. (Eds.) Eye movements: From physiology to cognition (pp. 181-190). Amsterdam: North-Holland.

Jürgens, R., Becker, W. \& Kornhuber, H. H. (1981). Natural and drug-induced variations of velocity and duration of human saccadic eye movements: Evidence for a control of the neural pulse generator by local feedback. Biological Cybernetics, 39, 87-96.

Kalesnykas, R. P. \& Hallett, P. E. (1987). The differentiation of visually guided and anticipatory saccades in gap and overlap paradigms. Experimental Brain Research, 68, 115-121.

Kowler, E. \& Steinman, R. M. (1979a). The effect of expectations on slow oculomotor control-I: Periodic target steps. Vision Research, 19, 619-632.

Kowler, E. \& Steinman, R. M. (1979b). The effect of expectations on slow oculomotor control II: Single target displacements. Vision Research, 19, 633-646.

Lee, C., Rohrer, W. H. \& Sparks, D. L. (1988). Population coding of saccadic eye movements by neurons in the superior colliculus. Nature, London, 332, 357-360.

Mcllwain, J. T. (1976). Large receptive fields and spatial transformations in the visual system. International Reviews Physiology, 10, 223-248.

Mcllwain, J. T. (1982). Lateral spread of neural excitation during microstimulation in intermediate gray layer of cat's superior colliculus. Journal of Neurophysiology, 47, $167-178$.

Ottes, F. P., Van Gisbergen, J. A. M. \& Eggermont, J. J. (1984). Metrics of saccade responses to visual double stimuli: Two different modes. Vision Research, 24, 1169-1179.

Ottes, F. P., Van Gisbergen, J. A. M. \& Eggermont, J. J. (1986). Visuomotor fields of the superior colliculus: A quantitative model. Vision Research, 26, 857-873.

Robinson, D. A. (1972). Eye movements evoked by collicular stimulation in the alert monkey. Vision Research, 12, 1795-1808.

Robinson, D. A. (1975). Ocular motor control signals. In Lennerstrand, G. \& Bach-y-Rita P. (Eds.) Basic mechanisms of ocular motility and their clinical implications (pp. 337-374). Oxford: Pergamon Press.

Schiller, P. H. \& Stryker, M. (1972). Single-unit recording and stimulation in superior colliculus of the alert rhesus monkey. Journal of Neurophysiology, 35, 915-924.

Schmidt, D., Abel. L. A., Dell'Osso, L. F. \& Daroff, R. B. (1979). Saccadic velocity characteristics: Intrinsic variability and fatigue. Aviation and Space Environmental Medicine, 50, 393-395. 
Sparks, D. L. (1986). Translation of sensory signals into commands for control of saccadic eye movements: Role of primate superior colliculus. Physiological Reviews, 66, 118-171.

Sparks, D. L. \& Mays, L. E. (1980). Movement fields of saccade-related burst neurons in the monkey superior colliculus. Brain Research, 190, 39-50.

Sparks, D. L. \& Mays. L. E. (1983). Spatial localization of saccade targets. I. Compensation for stimulation-induced perturbations in eye positions. Journal of Neurophysiology, 31, 659-669.

Sparks, D. L., Holland, R. \& Guthrie, B. L. (1976). Size and distribution of movement fields in the monkey superior colliculus. Brain Research, 113, 21-34.

Van Gisbergen, J. A. M. \& Van Opstal, A. J. (1987). Saccade endpoint scatter and properties of the collicular motor map. Society of Neuroscience Abstracts, 13, (Part 2), 1093.

Van Gisbergen, J. A. M., Robsinson, D. A. \& Gielen, S. (1981). A quantitative analysis of generation of saccadic eye movements by burst neurons. Journal of Neurophysiology, 45, 417-442.

Van Gisbergen, J. A. M., Van Opstal, A. J. \& Schoenmakers, J. J. M. (1985). Experimental test of two models for the generation of oblique saccades. Experimental Brain Research, 57, 321-336.

Van Gisbergen, J. A. M., Van Opstal, A. J. \& Tax, A. A. M. (1987). Collicular ensemble coding of saccades based on vector summation. Neuroscience, 21, 541-555.

Van Opstal, A. J. \& Van Gisbergen, J. A. M. (1987a). Nonhomogeneity and anisotropy in the topographical organization of the motor colliculus and saccade endpoint scatter. In Lüer, G. \& Lass, U. (Eds.) Proceedings of the fourth european conference on eye movements (pp. 114-116). Toronto: Hogrefe.

Van Opstal, A. J. \& Van Gisbergen, J. A. M. (1987b). Skewness of saccadic velocity profiles: A unifying parameter for normal and slow saccades. Vision Research, 27, 731-745.

Van Opstal, A. J. \& Van Gisbergen, J. A. M. (1989). A model for collicular efferent mechanisms underlying the generation of saccades. Brain, Behaviour and Evolution 30 , 90-94.

\section{APPENDIX}

In this section the following two consequences of the population jitter model will be discussed:

(1) the model yields an elliptical distribution of saccade vectors;

(2) the amount of scatter increases roughly linearly with eccentricity.

In this model we have assumed that the location jitter can be described by a rotation-symmetrical Gaussian function, with standard deviation $\sigma_{s}$. The $\sigma_{s}$-contour of this distribution is given by the circle:

$$
(\Delta u)^{2}+(\Delta v)^{2}=\left(\sigma_{s}\right)^{2}
$$

where $\Delta u$ and $\Delta v$ are small displacements in the Cartesian $u$ and $v$-directions of the collicular motor map. To explain how circular-symmetrical noise of this type at the level of the motor map is expressed in the scatter of the associated saccade vectors we need an expression which specifies how such a circle of population activity centers in the map is transformed into saccade vectors. This transformation from the collicular $(u, v)$ to the motor $(R, \Phi)$ domain is given, in general, by the Jacobian matrix in equation (A2):

$$
\left(\begin{array}{l}
\Delta u \\
\Delta v
\end{array}\right)=\left[\begin{array}{ll}
\partial u / \partial R & \frac{1}{R} \cdot \partial u / \partial \Phi \\
\partial v / \partial R & \frac{1}{R} \cdot \partial v / \partial \Phi
\end{array}\right] \cdot\left(\begin{array}{c}
\Delta R \\
R \cdot \Delta \Phi
\end{array}\right)
$$

The Jacobian matrix contains the partial derivatives $\partial u / \partial R, \partial v / \partial R$, etc. In our particular case, these derivatives can be computed from expressions for the inverse efferent mapping function of the motor colliculus, proposed by Ottes et al. (1986):

$$
\begin{array}{r}
\left(\begin{array}{l}
\Delta u \\
\Delta v
\end{array}\right)=\frac{1}{N} \cdot\left(\begin{array}{lr}
B_{u} \cdot(R+A \cdot \cos (\Phi)) & -B_{u} \cdot A \cdot \sin (\Phi) \\
B_{r} \cdot A \cdot \sin (\Phi) & B_{r} \cdot(R+A \cdot \cos (\Phi))
\end{array}\right) \\
\times\left(\begin{array}{c}
\Delta R \\
R \cdot \Delta \Phi
\end{array}\right) ;(\mathrm{A} 3)
\end{array}
$$

where $B_{u}, B_{i}$ and $A$ are the parameters of the complexlogarithmic mapping function which completely determine its shape (see Ottes et al., 1986, for more details) and $N=R^{2}+A^{2}+2 \cdot A \cdot R \cdot \cos (\Phi)$.

For simplicity, we will now compute the distribution of saccade vectors around the horizontal meridian $(\Phi=0)$. Equation (A3) then simply reduces to:

$$
\left(\begin{array}{l}
\Delta u \\
\Delta v
\end{array}\right)=\left(\begin{array}{lr}
B_{u} /(R+A) & 0 \\
0 & B_{r} /(R+A)
\end{array}\right) \cdot\left(\begin{array}{c}
\Delta R \\
R \cdot \Delta \Phi
\end{array}\right) .
$$

It is now straightforward to compute the distribution of saccade vectors by substitution of equation (A4) into equation (Al):

$$
\left(\frac{\Delta R}{B_{r}}\right)^{2}+\left(\frac{R \cdot \Delta \Phi}{B_{u}}\right)^{2}=\left(\frac{R+A}{B_{u} \cdot B_{r}}\right)^{2} \cdot\left(\sigma_{s}\right)^{2} .
$$

The implications of equation (A5) are twofold: first, the equation describes an ellipse with axes of symmetry along the $R$ and $\Phi$-directions. The length ratio of the two axes is given by the ratio $B_{r} / B_{u}$, which is the amount of anisotropy of the motor map. Second, equation (A5) states that the amount of scatter in the $(R, \Phi)$ domain is proportional to $R$ and implies that for $R \gg A$ is it approximately linearly related to $R$.

For the more general case $(\Phi \neq 0)$ one has to substitute equation (A3) into equation (A1) which yields a more complicated expression. However, for $R \gg A$ (in the model $A=3.0 \mathrm{deg}$ ) the ellipse retains the same characteristics as given by equation (A5).

Note, in Fig. 9, that the data for small amplitudes $(R=2$ and $R=5 \mathrm{deg}$ ) are clearly not rotation-symmetrical despite the fact that the value for scatter ratio $\sigma_{R} / \sigma_{\phi}$ is within the normal range (see Fig. 6A). In order, for the location jitter model to hold over the whole range of amplitudes, yielding rotation-symmetrical distributions in collicular coordinates in all cases, the scatter plots for small amplitudes should have been more elongated along the $R$-dimension [by about a factor of $1+(R / A)$, see equation (AS)] than we actually found in our data. 